Background Lower limb muscle strain is also a common injury in elite volleyball athletes and lead to sport absence. A proper assessment is crucial to understand injury risk and which factors we should modify to prevent it.

Objective To identify the contribution of lower limb strength, range of motion (ROM), alignment and functional stability on lower limb muscle strain in elite volleyball athletes.

Design Cross-sectional study.

Setting One volleyball team facility.

Patients (or Participants) Twenty-five male elite volleyball athletes.

Interventions (or Assessment of Risk Factors) All data were collected before the beginning of the competitive season. Athletes were aleatory selected in some stations with physiotherapists previously trained to apply the following tests: Shank-forefoot alignment, passive hip internal rotation ROM, Y-Test, hip extensors strength, knee flexors and extensors strength. Injuries were collected from the historic of past seasons.

Main Outcome Measurements Injuries were considered when the athlete had any muscle complaint and stayed absent from the sport for at least one training or match sessions.

Results 9 muscle strains were found, which represents $41 \%$ of all injuries on the team. The regression model revealed that hip extensor strength was associated with muscle strain in elite volleyball athletes $\left(F=8.050 ; r=0.518 ; R^{2}=0.235 ; p=\right.$ $0.010)$. Specifically, weakness of hip extensors increases the chance of lower limb muscle strain in elite volleyball athletes $(\mathrm{B}=-0.250)$.

Conclusions Hip extensor weakness explains 23\% of lower limb muscle strains in elite volleyball athletes. Prevention programs should include hip extensor strengthening to decrease the chance of lower limb muscle strain in elite volleyball athletes.

\section{KINEMATIC RISK FACTORS OF LOWER EXTREMITY OVERUSE INJURIES DURING LANDING TASKS IN A PHYSICALLY ACTIVE POPULATION: A SYSTEMATIC REVIEW AND META-ANALYSIS}

Camilla De Bleecker, Stefan Vermeulen, Cedric De Blaiser, Tine Willems, Roel De Ridder, Philip Roosen. Department of Rehabilitation Sciences, Faculty of Medicine and Health Sciences, Ghent University, Ghent, Belgium

\subsection{6/bjsports-2021-IOC.308}

Background Lower extremity overuse injuries are common in athletes participating in sports with repeated bouts of landing manoeuvres.

Objective To summarise and determine the relationship between kinematic alterations during a landing task and lower extremity overuse injuries in physically active populations.

Design Systematic review and meta-analysis considering prospective cohort, cross sectional or case-control study designs.

Setting Electronic databases PubMed, Embase, Web of Science, CINAHL, and SPORTDiscus were consulted in February 2020.

Participants Articles including an athletic or physically active, healthy population with an overuse injury of the lower extremity.

Assessment of risk factors Methodological quality was assessed by a modified Downs and Black checklist.

Main outcome measurements The relationship between threedimensional (3D) landing kinematics in physically active populations and lower extremity overuse injuries.
Results Twenty-three studies that investigated 3D landing kinematics in subjects with either patellar tendinopathy (PT), patellofemoral pain (PFP), exertional medial tibial pain (EMTP) or groin overuse injury met the inclusion criteria. Based on this systematic review, there is evidence for decreased knee flexion range of motion (ROM) and increased knee abduction ROM during landing as risk factors for PFP. For PT, risk factors are poorly understood. Furthermore, the meta-analysis demonstrated significantly greater hip adduction at initial contact (IC) $(p=0.02)$, greater knee internal rotation at IC $(p<0.001)$, greater peak knee external rotation $(p=0.05)$ and less ankle dorsiflexion at peak vertical ground reaction force (vGRF) $(p=0.05)$ in subjects with knee overuse injuries compared to healthy controls. There is evidence of increased trunk, hip and knee transversal ROM as risk factors for EMTP. Groin injuries are associated with greater pelvic and hip frontal and transversal plane ROM in the injured group compared to the healthy controls.

Conclusion The results of this systematic review and metaanalysis provide preliminary evidence for impaired landing kinematics associated with lower extremity overuse injuries. Excessive frontal and transversal plane movements during landing manoeuvres might increase impact and tensile forces resulting in lower extremity overuse injuries.

\section{ALTERATIONS IN WHOLE-BODY BIOMECHANICS DURING FAILED AND SUCCESSFUL UNANTICIPATED SINGLE-LEG LANDINGS: IMPLICATIONS FOR INJURY PREVENTION}

${ }^{1}$ Nicholas Romanchuk, ${ }^{1}$ Kenneth Smale, ${ }^{2}$ Michael Del Bel, 1,2,3 Daniel Benoit. 'School of Human Kinetics, University of Ottawa, Ottawa, Canada; ${ }^{2}$ School of Rehabilitation Sciences, University of Ottawa, Ottawa, Canada; ${ }^{3}$ Ottawa Carleton Institute for Biomedical Engineering, Ottawa, Canada

\subsection{6/bjsports-2021-IOC.309}

Background Non-contact knee injuries often occur when a stimulus alters decision-making during the flight phase of a landing task. We developed a novel protocol to study this paradigm.

Objective To evaluate biomechanical differences between failed and successful single-leg drop-jump landings requiring in-flight decision-making.

Design Cross-sectional study.

Setting Controlled laboratory setting.

Patients (or Participants) Thirty-two healthy male $(n=16 ; 15.9$ \pm 1.87 yrs.) and female $(15.7 \pm 1.7$ yrs.) competitive Ottawa area athletes with no history of musculoskeletal injury affecting functional performance.

Interventions (or Assessment of Risk Factors) Participants completed single-leg drop-jump landings from a platform aligned to their tibial plateau. The landing leg (left-right-both) was randomly assigned to the participant on a projector. Landings were categorized as 'successful' or 'failed' (defined as any loss of balance forcing a participant to adjust their base of support during landing).

Main Outcome Measurements Whole body kinematics (Vicon) and muscle excitation amplitudes (EMG) were normalized over the preparatory (flight) and reactive (landing) phases of the drop jump. Moving Average Convergence Divergence (MACD) analysed significant variables to identify when the differences began. 
Results Participants landed with more trunk flexion (success: $21.8^{\circ} \pm 13.2^{\circ}$; failed: $\left.30.3 \pm 17.2^{\circ}\right)(p<0.05)$, more anterior pelvic tilt (success: $4.2^{\circ} \pm 5.4$; failed: $7.7^{\circ} \pm 5.1^{\circ}$ ), and less lateral pelvic tilt towards the landing leg (success: $4.7 \pm 3.0^{\circ}$; failed: $2.7 \pm 3.7)$ during failed landings $(p<0.05)$. Higher rectus femoris, biceps femoris and gluteus medius excitation amplitudes were also observed during the failed landings $(p<0.05)$. MACD analysis identified that differences between failed and successful landings were initiated during the preparatory phase of the drop-jump.

Conclusions While biomechanical variables were significantly different during the landing phase, our novel MACD analysis identified that these differences initiated during the flight phase. Our findings also highlight that proximal joints play a critical role for landing successfully. Preventive training must therefore include how athletes prepare for a landing with a strong emphasis on upper body and proximal joint control.

\section{DOES HIP STRENGTH PREDICT DYNAMIC VALGUS IN FEMALE RECREATIONAL RUNNERS?}

1,2Gabriel Zeitoune, ${ }^{2} J u r a n d i r$ Nadal, ${ }^{3}$ Luiz Alberto Batista, 'Leonardo Metsavaht, ${ }^{4}$ Paulo Lucareli, 'Gustavo Leporace. 'Institute Brazil of Technologies in Health, RJ, Brazil; ${ }^{2}$ Biomedical Engineering Program, Federal University of Rio de Janeiro, RJ, Brazil; ${ }^{3}$ Biomechanics and Motor Behaviour Laboratory, State University of Rio de Janeiro, RJ, Brazil; ${ }^{4}$ Post Graduation Program of Rehabilitation Sciences, SP, Brazil

\subsection{6/bjsports-2021-IOC.310}

Background Dynamic valgus has been the focus of many studies to identify its association to an increased risk of runningrelated injuries. Many therapists suggest gluteus strengthening to address this movement dysfunction. However, it is not known which hip strength associated with this movement dysfunction.

Objective To test the correlation between hip strength and dynamic valgus in female runners.

Design Cross-sectional study.

Setting Biomechanics laboratory.

Participants Twenty-nine healthy recreational female runners.

Interventions Participants ran on a treadmill at $2.92 \mathrm{~m} / \mathrm{s}$. Kinematic data were collected using an 8 high-speed cameras motion analysis system (Vicon, Oxford, UK) with a sample rate of $250 \mathrm{~Hz}$. Maximal isometric hip strength was tested using a hand-held dynamometer.

Main Outcome Measurements Predictor variables were peak isometric strength of hip extensors, abductors and external rotators. Interest variables were contralateral pelvic drop, hip adduction and internal rotation (peak angles and joint excursion) during stance phase of running. Association between predictors and interest variables were tested using Pearson Correlation Coefficient (alpha $=0.05$ ).

Results There was no significant correlation between hip strength and contralateral pelvic drop ( $\mathrm{r}$ ranging from -0.09 to $0.32, \mathrm{p}>0.05$ ), hip adduction ( $\mathrm{r}$ ranging from -0.23 to $0.11, \mathrm{p}>0.05)$, and hip internal rotation ( $\mathrm{r}$ ranging from 0.33 to $0.01, \mathrm{p}>0.05)$.

Conclusions Although previous studies showed dynamic valgus was associated to hip weakness during single-leg squat and jump-landing tasks, the results of our study suggested that caution should be taken when linking hip disorders in female runners during running to posterolateral hip strength. These findings could be related to the linear nature of the statistical methods used to predict the biomechanical dysfunctions. It might be necessary to apply more robust techniques, as Artificial Neural Networks and Random Forests, to understand how physical variables interact to predict dynamic valgus in runners.

\section{SHORT TRACK VS HOCKEY HELMETS: INVESTIGATING IMPACT ATTENUATION PROPERTIES OF HELMETS IN TWO SKATING SPORTS}

${ }^{1}$ Daniel Aponte, ${ }^{2}$ Suzanne Leclerc, ${ }^{1}$ David Pearsall. 'McGill University, Montreal, Canada; ${ }^{2}$ Institut National du Sport du Québec, Montreal, Canada

\subsection{6/bjsports-2021-IOC.311}

Background Certification standards governing short track (ST) helmets only require high velocity impacts be tested. Rotational acceleration and low velocity impacts are mechanisms of injury which are known to cause concussion. Conversely, ice hockey (IH) helmet certification require low velocity impacts in addition to high velocity impacts, and have been designed to mitigate both impact velocities.

Objective To compare the impact attenuation characteristics between ST and IH helmets, in both high and low velocity impacts.

Design Two-group experimental design.

Setting Impacts were performed in laboratory under controlled conditions. Helmets were impacted at two impact velocities (high and low; $4.5 \mathrm{~m} / \mathrm{s}$ and $2.4 \mathrm{~m} / \mathrm{s}$ respectively) and four impact locations (rear, rear boss, side and front boss). This was performed using a linear impactor device and the Hybrid III surrogate headform and neck.

Patients (or Participants) 5 different helmet models; 3 ST models and 2 IH models.

Interventions (or Assessment of Risk Factors) Assessment of ST and $\mathrm{IH}$ helmet impact attenuation under various conditions.

Main Outcome Measurements Peak linear and rotational acceleration; Head Injury Criterion (HIC) and Brain Injury Criterion (BrIC).

Results Between-groups ANOVA for linear [Low $F(1,27)=$ 10.7, $\mathrm{p}<0.05, \eta 2=0.284$; High $=\mathrm{F}(1,24)=5.8, \mathrm{p}<0.05$, $\eta 2=0.195]$ and rotational [Low $\mathrm{F}(1,27)=15.8, \mathrm{p}<0.05$, $\eta 2=0.370 ;$ High $=F(1,24)=8.1, p<0.05, \eta 2=0.251]$ accelerations yielded statistically significant differences with large effect sizes for all impact locations in both impact velocities. One-way between-helmet ANOVAs and post-hoc Bonferroni revealed impact attenuation performance hierarchy: IH 2 $>\mathrm{IH} 1>\mathrm{ST} 3>\mathrm{ST} 1>\mathrm{ST} 2$. Between-groups ANOVA revealed statistical differences for HIC [Low $\mathrm{F}(1,27)=14.1$, $\mathrm{p}<0.05, \eta 2=0.344 ;$ High $=\mathrm{F}(1,24)=7.6, \mathrm{p}<0.05, \eta 2=$ $0.241]$. BrIC results were mixed.

Conclusions Results suggest that these IH helmets are better at attenuating both impact velocities than this group of ST helmets. Interestingly, the largest effect sizes were observed in the low-velocity impacts.

\section{DO MOUTHGUARDS PREVENT ORO-FACIAL TRAUMA IN SPORT: A SYSTEMATIC REVIEW}

Peter Fine. UCL Eastman Dental Institute, London, UK

10.1136/bjsports-2021-IOC.312 